\title{
AN APPROXIMATION PROPERTY FOR TEICHMÜLLER POINTS
}

\author{
Alexandru Buium
}

Start with a field $K$ of characteristic zero, complete under a discrete valuation and having an algebraically closed residue field $k$ of characteristic $p>0$. Let $R$ be the valuation ring of $K$. Assume that we are given a prime element $\pi \in R$ which is algebraic over $Q_{p}$. Let $q$ be the cardinality of the residue field of $Q_{p}(\pi)$ and let $\phi$ be the unique ring automorphism of $R$ with $\phi(\pi)=\pi$ that lifts the "Frobenius" automorphism $F: k \rightarrow k, F(x):=x^{q}$.

Let $X$ be a scheme of finite type over $R$ and assume the Frobenius $x \mapsto x^{q}$ of the closed fibre $X_{0}:=X \otimes k$ lifts to a $\phi$-endomorphism $\tilde{\phi}$ of $\hat{X}$, the completion of $X$ with respect to the ideal generated by $\pi$. For any point $P \in X(R)=\hat{X}(R), P: \operatorname{Spf} R \rightarrow \hat{X}$ define the point $P^{\tilde{\phi}} \in X(R)$ as the composition

$$
\operatorname{Spf} R \stackrel{\phi^{-1}}{\rightarrow} \operatorname{Spf} R \stackrel{P}{\rightarrow} \hat{X} \stackrel{\tilde{\phi}}{\rightarrow} \hat{X}
$$

Call $P$ a Teichmüller point if $P^{\tilde{\phi}}=P$ and let $T=T(X, \tilde{\phi}) \subset X(R)$ denote the set of Teichmüller points.

\section{Examples}

1) Let $X=\left(G_{m}\right)^{N}=$ Spec $R\left[x_{1}, x_{1}^{-1}, \ldots, x_{N}, x_{N}^{-1}\right]$ be a torus over $R$ and let $\tilde{\phi}$ be the unique lifting of $\phi$ to $\hat{X}$ such that $\tilde{\phi}\left(x_{i}\right)=x_{i}^{q}+\pi g_{i}$, where $g_{i} \in R\left[x_{1}, \ldots, x_{N}\right]$. So $T$ consists of all points $r=\left(r_{1}, \ldots, r_{N}\right) \in R^{\times} \times \ldots \times R^{\times}$ such that $\phi\left(r_{i}\right)=r_{i}^{q}+\pi g_{i}(r)$. So if $K$ is the completion of the maximum unramified extension of $Q_{p}$ and $g_{i}=0$, then $T$ consists all points whose coordinates are roots of unity of order prime to $p$.

2) Assume for simplicity that $K$ is the completion of the maximum unramified extension of $Q_{p}$. Let $X$ be an abelian variety with ordinary reduction $X_{0}$ and assume $X$ is the canonical lifting of $X_{0}$; by [Ka1], there is a canonical lifting of Frobenius, $\tilde{\phi}$, to $\hat{X}$. Then $T$ contains the prime to $p$ torsion of $X(R)$.

Received April 1, 1996.

Supported in part by NSF grant DMS-9500331. 
3) Assume again that $K$ is as in Example 2) above. Let $n \geq 3$ be an integer not divisible by $p$. Let

$$
X=\operatorname{Spec}_{\bar{M}_{n} \otimes R}\left(\operatorname{Symm}\left(\omega^{p-1}\right) /\left(E_{p-1}-1\right)\right)
$$

where $\bar{M}_{n}$ is obtained by "adding cusps" to the modular scheme over $Z[1 / n]$ classifying the elliptic curves with level $n$ structure (cf. [Ka2], pp. $81-82), \omega$ is the natural invertible sheaf defined in [Ka2], p. 82, and $E_{p-1}$ is the corresponding Eisenstein series. By [Ka2], p.111, $X$ is an affine scheme. By [Ka2] pp. 122-124, there is a natural lifting of the Frobenius, $\tilde{\phi}$, to $\hat{X}$. Using the construction of $\tilde{\phi}$ via the "canonical subgroup" one can check that $T$ contains all points of $X(R)$ that are not cusps and for which the corresponding elliptic curve $E / R$ is a canonical lift of its closed fibre $E_{0}$. One can consider more complicated examples by taking $d$ fold products of the $X$ above. Also it is reasonable to expect that a similar example is obtainable by taking modular varieties corresponding to abelian varieties of higher dimension.

4) Let $X=\operatorname{Proj} R\left[x_{0}, \ldots, x_{N}\right]$ be the projective space over $R$ and let $\tilde{\phi}$ be the unique lifting of $\phi$ to $\hat{X}$ such that

$$
\tilde{\phi}\left(\frac{x_{i}}{x_{j}}\right)=\frac{x_{i}^{q}+\pi G_{i}}{x_{j}^{q}+\pi G_{j}}
$$

where $G_{0}, \ldots, G_{N} \in R\left[x_{0}, \ldots, x_{N}\right]$ are homogenous polynomials of degree $q$. So, for instance, if $G_{i}=0$ and $K$ is the completion of the maximum unramified extension of $Q_{p}$ then $T$ consists all points whose projective coordinates have ratios roots of unity of order prime to $p$.

Let $X / R$ be a scheme, let $Y \subset X$ be a closed subscheme and let $P \in$ $X(R)$. Set $R_{m}:=R / \pi^{m+1} R$ for $m \geq 1, X_{m}:=X \otimes R_{m}, Y_{m}:=Y \otimes R_{m}$, and let $P_{m} \in X_{m}\left(R_{m}\right)$, be the image of $P$. One defines the $p$-adic distance from $P$ to $Y$ as

$$
\operatorname{dist}(P, Y)=\inf \left\{p^{-m} ; P_{m} \in Y_{m}\left(R_{m}\right)\right\} .
$$

Of course $\operatorname{dist}(P, Y)=0$ if and only if $P \in Y(R)$. Here is our main result:

Theorem 1. Let $X / R$ be a scheme of finite type, $Y \subset X$ a closed subscheme, $\tilde{\phi}: \hat{X} \rightarrow \hat{X}$ a $\phi$-lifting of the Frobenius of the closed fibre $X_{0}$ and $T=T(X, \tilde{\phi}) \subset X(R)$ the set of Teichmüller points. Then there exists a real constant $c=c(X, Y, \tilde{\phi})>0$ such that for any $P \in T$ with $\operatorname{dist}(P, Y) \leq c$ we must have $P \in Y(R)$.

Remark. Theorem 1 applied to Examples 1, 2, 3 above answers special cases of a question posed to the author by F.Voloch. Cf. [TV] for the case of Example 1. 
For the case of curves in projective varieties we can supplement the above Theorem as follows:

Theorem 2. Assume we are in the situation of Theorem 1 and assume moreover that $K$ is absolutely unramified, $X / R$ is projective and $Y / R$ is a smooth curve of genus $\geq 2$. Then the set of points $\{P \in T ; \operatorname{dist}(P, Y)<1\}$ is finite.

The proofs of these two results will be an easy consequence of a construction made in [B1] whose properties we now recall. For any $R$-algebra $B$ we denote by $W_{2}^{\pi}(B)$ the ring of "ramified Witt vectors of length two", whose underlying set is $B \times B$ and whose addition and multiplication are given by:

$$
\begin{gathered}
\left(b_{0}, b_{1}\right)+\left(c_{0}, c_{1}\right)=\left(b_{0}+c_{0}, b_{1}+c_{1}-(p / \pi) C_{q}\left(b_{0}, c_{0}\right)\right) \\
\left(b_{0}, b_{1}\right) \cdot\left(c_{0}, c_{1}\right)=\left(b_{0} c_{0}, b_{0}^{q} c_{1}+c_{0}^{q} b_{1}+\pi b_{1} c_{1}\right)
\end{gathered}
$$

where $C_{q}(X, Y)=\left((X+Y)^{q}-X^{q}-Y^{q}\right) / p \in Z[X, Y]$. Let $f: A \rightarrow B$ be an $R$-algebra homomorphism. By a $\pi$-derivation of $f$ we shall understand a map of sets $\delta: A \rightarrow B$ such that the induced map

$$
(f, \delta): A \rightarrow B \times B=W_{2}^{\pi}(B), \quad x \mapsto(f(x), \delta(x))
$$

is a ring homomorphism. For instance the map $\delta_{*}: R \rightarrow R$ defined by $\delta_{*} x=\left(\phi(x)-x^{q}\right) / \pi$ is a $\pi$-derivation. There is an obvious notion of $\pi$-derivation of a map of sheaves of $R$-algebras on a topological space. For any scheme of finite type $X / R$ we constructed, in [B1], a projective system

$$
\ldots \rightarrow X^{n} \stackrel{f_{n}}{\rightarrow} X^{n-1} \rightarrow \ldots \rightarrow X^{1} \stackrel{f_{1}}{\rightarrow} X^{0}=\hat{X}
$$

of $\pi$-formal schemes (where " $\pi$-formal scheme" means "formal scheme for which the ideal generated by $\pi$ is an ideal of definition"), and $\pi$-derivations $\delta_{n}$ (extending $\delta_{*}$ ) of $\mathcal{O}_{X_{n-1}}$ into the direct image of $\mathcal{O}_{X_{n}}$, such that each $\delta_{n+1}$ prolongs $\delta_{n}$ and such that the following universality property is satisfied. For any morphism of $\pi$-formal schemes $g: S \rightarrow X^{n}$ and for any $\pi$-derivation $\delta$ of $\mathcal{O}_{X^{n}}$ into $g_{*} \mathcal{O}_{S}$, prolonging $\delta_{n}$, there is a unique morphism of $\pi$-formal schemes $f: S \rightarrow X^{n+1}$ such that $f^{*} \circ \delta_{n+1}=\delta$ and $f_{n+1} \circ f=g$. This universality property induces natural maps

$$
\nabla^{n}: X(R) \rightarrow X^{n}(R)
$$

which induce bijections

$$
\nabla_{0}^{n}: X_{n}\left(R_{n}\right) \rightarrow X_{0}^{n}(k)
$$


where, as usual, $X_{0}^{n}:=X^{n} \otimes k$. (In case $K$ is absolutely unramified, but only in this case, the $k$-schemes $X_{0}^{n}$ are the "Greenberg transforms" of $X$.)

Now we are in a position to prove Theorem 1 . We may assume $\hat{X} / R$ is flat, hence we may define a $\pi$-derivation of the structure sheaf of $\hat{X}$ by the formula $\delta x=\left(\tilde{\phi}(x)-x^{q}\right) / \pi$. By the universality property of $X^{n}$, since the $\delta_{n}$ 's extend $\delta_{*}$, there exist induced sections $s^{n}: \hat{X} \rightarrow X^{n}$ of the projections $X^{n} \rightarrow \hat{X}$ such that $f_{n} \circ s^{n}=s^{n-1}$ for all $n$. Tensorizing with $k$ we get a system of sections $s_{0}^{n}: X_{0} \rightarrow X_{0}^{n}$ of the projections $X_{0}^{n} \rightarrow X_{0}$. Consider the closed subschemes $Y^{n} \subset X^{n}$ and their reductions modulo $p$, $Y_{0}^{n} \subset X_{0}^{n}$. Consider the closed subschemes

$$
Z(n)=\left(s_{0}^{n}\right)^{-1}\left(Y_{0}^{n} \cap s_{0}^{n}\left(X_{0}\right)\right) \subset X_{0} .
$$

They form a descending sequence so there exists an index $n_{0}$ such that

$$
\text { (*) } Z(n)=Z\left(n_{0}\right), \quad n \geq n_{0} .
$$

Now let $P \in T$, viewed as a morphism $P: S p f R \rightarrow \hat{X}$. We claim that $\nabla^{n}(P): S p f R \rightarrow X^{n}$ factors through $s^{n}(\hat{X})$, i.e. that $\nabla^{n}(P)=s^{n} \circ P$. (Indeed we proceed by induction on $n$. Assume $\nabla^{n-1}(P)=s^{n-1} \circ P$. Since $P \in T$ we have $P^{*} \circ \delta=\delta_{*} \circ P^{*}$. On the other hand, by the construction of the $s^{n}$ 's we have $s^{n, *} \circ \delta_{n}=\delta \circ s^{n-1, *}$. We get

$$
\begin{gathered}
\left(s^{n} \circ P\right)^{*} \circ \delta_{n}=P^{*} \circ s^{n, *} \circ \delta_{n}=P^{*} \circ \delta \circ s^{n-1, *} \\
=\delta_{*} \circ P^{*} \circ s^{n-1, *}=\delta_{*} \circ \nabla^{n-1}(P)^{*} .
\end{gathered}
$$

On the other hand, by the definition of $\nabla^{n}$ we have

$$
\nabla^{n}(P)^{*} \circ \delta_{n}=\delta_{*} \circ \nabla^{n-1}(P)^{*} .
$$

The two equations above plus the universality property of $X^{n}$ impliy that $\nabla^{n}(P)=s^{n} \circ P$, and the induction step is proved.) We conclude that $\nabla_{0}^{n}\left(P_{n}\right) \in s_{0}^{n}\left(X_{0}\right)(k)$ for all $\mathrm{n}$. In particular if $P \in T$ and $\operatorname{dist}(P, Y) \leq$ $p^{-n_{0}}$ we get $\nabla_{0}^{n_{0}}\left(P_{n_{0}}\right) \in Y_{0}^{n_{0}}(k) \cap s_{0}^{n_{0}}\left(X_{0}\right)(k)$, hence, by $(*), \nabla_{0}^{n}\left(P_{n}\right) \in$ $Y_{0}^{n}(k) \cap s_{0}^{n}\left(X_{0}\right)(k)$ for all $n \geq n_{0}$, hence $P \in Y(R)$ and we are done.

To prove Theorem 2 it is enough to prove that $Y_{0}^{1} \cap s_{0}^{1}\left(X_{0}\right)$ is a finite set. But $s_{0}^{1}\left(X_{0}\right)$ is a projective variety while, by [B2], Proposition 1.10, $Y_{0}^{1}$ is an affine variety. Since both these varieties are closed in $X_{0}^{1}$, their intersection must be finite.

\section{Acknowledgment}

The author would like to thank John Tate for his useful comments. 


\section{References}

[B1] A. Buium, Differential characters of abelian varieties over $p$-adic fields, Invent. Math. 122 (1995), 309-340.

[B2] _ _ Geometry of p-jets, Duke Math. J. 82 (1996), 349-367.

[Ka1] N. Katz, Serre-Tate local moduli, Springer, LNM 868 (1981), 138-202

[Ka2] N. Katz, p-adic properties of modular schemes and modular forms, in: Modular functions in one variable III, Springer, LNM 350 (1973), 70-190.

[TV] J. Tate and F.Voloch, Projective geometry for $p$-adic fields, Preprint.

University of New Mexico, Albuquerque, NM 87131

E-mail address: buium@math.unm.edu 